\title{
The optimum expression for the gravitational potential of polyhedral bodies having a linearly varying density distribution
}

\author{
Hamayun - I. Prutkin • R. Tenzer
}

Received: 16 November 2007 / Accepted: 1 July 2009 / Published online: 26 July 2009

(C) The Author(s) 2009. This article is published with open access at Springerlink.com

\begin{abstract}
When topography is represented by a simple regular grid digital elevation model, the analytical rectangular prism approach is often used for a precise gravity field modelling at the vicinity of the computation point. However, when the topographical surface is represented more realistically, for instance by a triangular irregular network (TIN) model, the analytical integration using arbitrary polyhedral bodies (the analytical line integral approach) can be implemented directly without additional data pre-processing (gridding or interpolation). The analytical line integral approach can also facilitate 3-D density models created for complex geometrical bodies. For the forward modelling of the gravitational field generated by the geological structures with variable densities, the analytical integration can be carried out using polyhedral bodies with a varying density. The optimal expression for the gravitational attraction vector generated by an arbitrary polyhedral body having a linearly varying density is known. In this article, the corresponding optimal expression for the gravitational potential is derived by means of line integrals after applying the Gauss divergence theorem.
\end{abstract}

Keywords Gravitational potential · Line integral · Linear density · Polyhedron

Hamayun ( $\varangle) \cdot$ I. Prutkin

Delft Institute of Earth Observation and Space Systems (DEOS),

TU Delft, Kluyverweg 1, 2629 HS Delft, The Netherlands

e-mail: hamayunkhan2000@yahoo.com

R. Tenzer

Faculty of Sciences, School of Surveying,

University of Otago, 310 Castle Street, Dunedin, New Zealand

\section{Introduction}

Various methods are applied to evaluate Newton's volume integral. Studying the local gravity field, a simple form of the integration volume can be used, such as the right rectangular parallelepiped (prism) with constant density within each individual integration volume. Bessel (1813) derived the closed analytical expression for the potential of a prism. The potential-related formulae for a prism were studied also by Zach (1811), Mollweide (1813), Everest (1830) and Mader (1951). More recently, Nagy et al. (2000) summarized the closed analytical expressions for the potential and its first and second derivatives of a rectangular prism of homogenous density. However, in most geological structures the constant density assumption does not hold. For this reason, some authors derived analytical expressions for volume elements with linearly or otherwise varying density distribution models. Chai and Hinze (1988) computed gravity anomalies using a rectangular prism with density changing linearly with depth. Gallardo-Delgado et al. (2003) derived the analytical solution for the forward gravity modelling utilizing a right rectangular prism with density varying according to a polynomial quadratic law. García-Abdeslem $(1992,2005)$ introduced the analytical expression for the right rectangular prism with depth dependent density distribution having a form of a cubic polynomial.

For the gravity field modelling of inhomogeneous density formations, the approximation of geological structures by more general geometrical forms than rectangular prisms are implemented. Hurbbert (1948) introduced a methodology called the line integral approach; the surface or volume integrals are converted to line integrals after applying the Gauss divergence theorem. Following this idea, Talwani et al. (1959) applied the line integral approach to the polygon in 2-D. Talwani and Ewing (1960), Collette (1965) and 
Takin and Talwani (1966) decomposed the 3-D body into parallel, typically horizontal laminae. Paul (1974) and Barnett (1976) generalized this concept for a polyhedron in 3-D. Pohánka (1988) derived a simple algorithm for the attraction of a homogeneous polyhedral body using the line integral approach (see also Ivan 1990; Pohánka 1990). The formulae for polyhedral bodies with homogeneous density were studied also by Okabe (1979), Götze and Lahmeyer (1988), Kwok (1991), Holstein and Ketteridge (1996), Werner and Scheeres (1997), Holstein et al. (1999) and Holstein (2002a,b). Petrović (1996) presented in more complete form the formulae for the potential and its derivatives using the line integral approach for arbitrary polyhedral bodies of homogenous density (see also Tsoulis and Petrović 2001)

In forward modelling of the gravitational field of geological structures with the variable density distribution, the analytical expressions for volume elements with linearly or otherwise varying density distribution models improve the numerical efficiency. One example can be given in modeling the gravitational contribution of sedimentary basins where the density increases with depth due to compaction (e.g. Artemjev et al. 1994). Combining the benefits of using more generalized geometrical bodies and taking into account density variation models, Pohánka (1998) introduced the expression for the attraction of an arbitrary polyhedral body having a linearly varying density by means of line integrals. The alternative expression was derived by Hansen (1999). Holstein (2003) generalized their work deriving the formulae also for the potential and its second derivatives. To avoid singular terms and obtain a maximal numerical efficiency, Pohánka (1998) in his study derived the optimum expression and proposed a simple computational algorithm for computing the gravitational attraction.

In gravimetric geoid modelling and related subjects not only the attraction-related term (direct effect) but also the potential-related terms (primary and secondary indirect effects) are computed. Following the concept used by Pohánka $(1988,1998)$ in this study we derive the optimum expression for computing the gravitational potential of an arbitrary polyhedral body having a linearly varying density. The optimum expression for the gravitational potential of the polyhedral body having a homogeneous density is given in Pohánka (1988). Our derivation is thus reduced to find the optimum expression only for the linear density variation term. In comparison to the formula for the potential given by Holstein (2003), the main advantage of adopting the computational strategy developed by Pohánka $(1988,1998)$ is that our expression does not have the singular terms. We also demonstrate that the computational algorithm for computing the attraction proposed by Pohánka (1998) can directly be applied for computing the potential. The potential of an arbitrary polyhedral body having a linearly varying density by means of line integrals is derived in Sect. 2. The optimum expression and algorithm for computing the potential are provided in Sects. 3 and 4. The concluding remarks are given in Sect. 5.

\section{Potential by means of line integrals}

The gravitational potential $V$ of the polyhedral body at the point $\mathbf{r}$ is defined by the Newton volume integral

$V(\mathbf{r})=G \iiint_{D} \frac{\rho\left(\mathbf{r}^{\prime}\right)}{\left|\mathbf{r}^{\prime}-\mathbf{r}\right|} \mathrm{d} \tau^{\prime}$,

where $G$ is the gravitational constant, $\rho$ the mass density distribution function inside the volume $D$ of the polyhedral body, $\mathrm{d} \tau^{\prime}$ the volume element at the point $\mathbf{r}^{\prime}$, and $\left|\mathbf{r}^{\prime}-\mathbf{r}\right|$ the Euclidean spatial distance between the computation point $\mathbf{r}$ and the running integration point $\mathbf{r}^{\prime}$. Let us consider the linearly varying density (Pohánka 1998)

$\rho\left(\mathbf{r}^{\prime}\right)=\rho_{0}+\boldsymbol{\rho}_{\mathbf{1}} \cdot \mathbf{r}^{\prime}$,

where $\rho_{0}$ is the value of density at a suitably chosen origin of the local coordinate system used for a description of the density model within the volume $D$ of the polyhedral body, and $\boldsymbol{\rho}_{\mathbf{1}}$ the gradient of a linear density distribution function. Combining Eqs. (1) and (2), we get

$$
\begin{aligned}
V(\mathbf{r})= & G\left(\rho_{0}+\rho_{\mathbf{1}} \cdot \mathbf{r}\right) \iiint_{D} \frac{1}{\left|\mathbf{r}^{\prime}-\mathbf{r}\right|} \mathrm{d} \tau^{\prime} \\
& +G \boldsymbol{\rho}_{\mathbf{1}} \cdot \iiint_{D} \frac{\mathbf{r}^{\prime}-\mathbf{r}}{\left|\mathbf{r}^{\prime}-\mathbf{r}\right|} \mathrm{d} \tau^{\prime} .
\end{aligned}
$$

Using the identities:

$\nabla_{\mathbf{r}^{\prime}} \cdot \frac{\mathbf{r}^{\prime}-\mathbf{r}}{\left|\mathbf{r}^{\prime}-\mathbf{r}\right|}=\frac{2}{\left|\mathbf{r}^{\prime}-\mathbf{r}\right|}, \quad \nabla_{\mathbf{r}^{\prime}}\left|\mathbf{r}^{\prime}-\mathbf{r}\right|=\frac{\mathbf{r}^{\prime}-\mathbf{r}}{\left|\mathbf{r}^{\prime}-\mathbf{r}\right|}$,

Eq. (3) is further rewritten as

$$
\begin{aligned}
V(\mathbf{r})= & G \frac{\rho_{0}+\boldsymbol{\rho}_{\mathbf{1}} \cdot \mathbf{r}}{2} \iiint_{D} \nabla_{\mathbf{r}^{\prime}} \cdot \frac{\mathbf{r}^{\prime}-\mathbf{r}}{\left|\mathbf{r}^{\prime}-\mathbf{r}\right|} \mathrm{d} \tau^{\prime} \\
& +G \boldsymbol{\rho}_{\mathbf{1}} \cdot \iiint_{D} \nabla_{\mathbf{r}^{\prime}}\left|\mathbf{r}^{\prime}-\mathbf{r}\right| \mathrm{d} \tau^{\prime}
\end{aligned}
$$

To convert the volume integrals on the right-hand side of Eq. (4) to the surface integrals, the Gauss divergence theorem is applied. If $\mathbf{f}\left(\mathbf{r}^{\prime}\right)$ is a vector function with integrable gradient in the domain $D$, it holds

$$
\iiint_{D} \nabla_{\mathbf{r}^{\prime}} \cdot \mathbf{f}\left(\mathbf{r}^{\prime}\right) \mathrm{d} \tau^{\prime}=\oiint_{S} \mathbf{f}\left(\mathbf{s}^{\prime}\right) \cdot \mathrm{d} \boldsymbol{\sigma}^{\prime},
$$

where the surface element vector $\mathrm{d} \boldsymbol{\sigma}^{\prime}$ is defined as the product of the unit normal vector $\mathbf{n}\left(\mathbf{s}^{\prime}\right)$ oriented outwards from 
the volume $D$ and the scalar surface element $\mathrm{d} \sigma^{\prime}$ at the point $\mathbf{s}^{\prime}$ on the surface $S$, i.e. d $\boldsymbol{\sigma}^{\prime}=\mathbf{n}\left(\mathbf{s}^{\prime}\right) \mathrm{d} \sigma^{\prime}$. Correspondingly, if $f\left(\mathbf{r}^{\prime}\right)$ is a scalar function with integrable gradient in the domain $D$, the Gauss divergence theorem holds

$$
\iiint_{D} \nabla_{\mathbf{r}^{\prime}} f\left(\mathbf{r}^{\prime}\right) \mathrm{d} \tau^{\prime}=\oiint_{S} f\left(\mathbf{s}^{\prime}\right) \mathrm{d} \boldsymbol{\sigma}^{\prime} .
$$

The application of the Gauss divergence theorem in Eq. (4) yields

$$
\begin{aligned}
V(\mathbf{r})= & G \frac{\rho_{0}+\boldsymbol{\rho}_{\mathbf{1}} \cdot \mathbf{r}}{2} \oiint_{S} \frac{\mathbf{s}^{\prime}-\mathbf{r}}{\left|\mathbf{s}^{\prime}-\mathbf{r}\right|} \cdot \mathrm{d} \boldsymbol{\sigma}^{\prime} \\
& +G \boldsymbol{\rho}_{\mathbf{1}} \cdot \oiint_{S}\left|\mathbf{s}^{\prime}-\mathbf{r}\right| \mathrm{d} \boldsymbol{\sigma}^{\prime},
\end{aligned}
$$

where $\left|\mathbf{s}^{\prime}-\mathbf{r}\right|$ is the Euclidean spatial distance between the computation point $\mathbf{r}$ and the running integration point $\mathbf{s}^{\prime}$ on the surface $S$.

We further define the surface integrals on the right-hand side of Eq. (5) as a sum of the surface integrals over the polyhedral faces $\left\{S_{k}: k=1,2, \ldots, K\right\}$, where $K$ is the total number of the polyhedral faces. At any surface point $\mathbf{s}^{\prime}$ of the $k$-th polyhedral face $S_{k}$ we have $\mathrm{d} \boldsymbol{\sigma}^{\prime}=\mathbf{n}_{k} \mathrm{~d} \sigma^{\prime}$, where $\mathbf{n}_{k}$ is the unit normal vector oriented outwards from the polyhedral face $S_{k}$. Hence,

$$
\begin{aligned}
V(\mathbf{r})= & G \frac{\rho_{0}+\boldsymbol{\rho}_{\mathbf{1}} \cdot \mathbf{r}}{2} \sum_{k=1}^{K} \iint_{S_{k}} \frac{\mathbf{s}^{\prime}-\mathbf{r}}{\left|\mathbf{s}^{\prime}-\mathbf{r}\right|} \cdot \mathbf{n}_{k} \mathrm{~d} \sigma^{\prime} \\
& +G \sum_{k=1}^{K} \boldsymbol{\rho}_{\mathbf{1}} \cdot \iint_{S_{k}} \mathbf{n}_{k}\left|\mathbf{s}^{\prime}-\mathbf{r}\right| \mathrm{d} \sigma^{\prime} \\
= & G \sum_{k=1}^{K}\left[\frac{\rho_{0}+\boldsymbol{\rho}_{\mathbf{1}} \cdot \mathbf{r}}{2} \mathbf{n}_{k} \cdot \iint_{S_{k}} \frac{\mathbf{s}^{\prime}-\mathbf{r}}{\left|\mathbf{s}^{\prime}-\mathbf{r}\right|} \mathrm{d} \sigma^{\prime}\right. \\
& \left.+\boldsymbol{\rho}_{\mathbf{1}} \cdot \mathbf{n}_{k} \iint_{S_{k}}\left|\mathbf{s}^{\prime}-\mathbf{r}\right| \mathrm{d} \sigma^{\prime}\right] .
\end{aligned}
$$

In accordance with Pohánka (1998, Eq. 11), we denote

$$
\mathbf{G}_{k}(\mathbf{r})=\iint_{S_{k}} \frac{\mathbf{s}^{\prime}-\mathbf{r}}{\left|\mathbf{s}^{\prime}-\mathbf{r}\right|} \mathrm{d} \sigma^{\prime}
$$

and introduce

$$
H_{k}(\mathbf{r})=\iint_{S_{k}}\left|\mathbf{s}^{\prime}-\mathbf{r}\right| \mathrm{d} \sigma^{\prime}
$$

The gravitational potential $V$ in Eq. (6) then takes the following form

$$
V(\mathbf{r})=\mathrm{G} \sum_{k=1}^{K}\left[\frac{\rho_{0}+\boldsymbol{\rho}_{\mathbf{1}} \cdot \mathbf{r}}{2} \mathbf{n}_{k} \cdot \mathbf{G}_{k}(\mathbf{r})+\boldsymbol{\rho}_{\mathbf{1}} \cdot \mathbf{n}_{k} H_{k}(\mathbf{r})\right] .
$$

The solution of the surface integral in the vector function $\mathbf{G}_{k}(\mathbf{r})$ was derived by Pohánka (1998). We use a similar procedure for finding a closed analytical solution of the surface integral in the scalar function $H_{k}(\mathbf{r})$. Firstly, we apply the Gauss divergence theorem for converting the surface integral to a sum of line integrals along the closed polygon $L_{k}$ which forms the boundary of the polyhedral face $S_{k}$. If $\mathbf{h}_{k}\left(\mathbf{s}^{\prime}\right)$ is a vector function with integrable gradient in the domain $S_{k}$, and $\mathbf{n}_{k} \cdot \mathbf{h}_{k}\left(\mathbf{s}^{\prime}\right)=0$ (i.e. the vector $\mathbf{h}_{k}\left(\mathbf{s}^{\prime}\right)$ lies in the plane of the polyhedral face $S_{k}$ ), it holds

$$
\iint_{S_{k}} \nabla_{\mathbf{s}^{\prime}} \cdot \mathbf{h}\left(\mathbf{s}^{\prime}\right) \mathrm{d} \sigma^{\prime}=\oint_{L_{k}} \mathbf{h}\left(\mathbf{I}^{\prime}\right) \cdot \mathrm{d} \boldsymbol{\xi}^{\prime},
$$

where the line element vector $\mathrm{d} \boldsymbol{\xi}^{\prime}$ at the point $\mathbf{I}^{\prime}$ on the curve $L_{k}$ is orthogonal to the curve $L_{k}$ and to the vector $\mathbf{n}_{k}$, and it is oriented outwards from the domain $S_{k}$. To convert the surface integral in Eq. (8) to a sum of line integrals, we have to find the vector function $\mathbf{h}_{k}\left(\mathbf{s}^{\prime}\right)$ in the domain $S_{k}$ which satisfies the following two conditions:

$\nabla_{\mathbf{s}^{\prime}} \cdot \mathbf{h}_{k}\left(\mathbf{s}^{\prime}\right)=\left|\mathbf{s}^{\prime}-\mathbf{r}\right|$,

and

$\mathbf{n}_{k} \cdot \mathbf{h}_{k}\left(\mathbf{s}^{\prime}\right)=0$.

For this purpose, we decompose the vector $\mathbf{s}^{\prime}-\mathbf{r}$ into two subcomponents; the first component $\left(\mathbf{s}^{\prime}-\mathbf{r}\right)_{\|}$is parallel to the polyhedral face $S_{k}$, and the second component $\left(\mathbf{s}^{\prime}-\mathbf{r}\right)_{\perp}$ is perpendicular to the polyhedral face $S_{k}$; i.e.,

$$
\begin{aligned}
\left(\mathbf{s}^{\prime}-\mathbf{r}\right)_{\perp} & =Z_{k} \mathbf{n}_{\mathbf{k}}, \quad Z_{k}=\mathbf{n}_{\mathbf{k}} \cdot\left(\mathbf{s}^{\prime}-\mathbf{r}\right), \\
\left(\mathbf{s}^{\prime}-\mathbf{r}\right)_{\|} & =\mathbf{s}^{\prime}-\mathbf{r}-\left(\mathbf{s}^{\prime}-\mathbf{r}\right)_{\perp} \\
& =\mathbf{s}^{\prime}-\mathbf{r}-\mathbf{n}_{k} \mathbf{n}_{k} \cdot\left(\mathbf{s}^{\prime}-\mathbf{r}\right) .
\end{aligned}
$$

It follows from the second condition (cf. Eq. (12) that the vector $\mathbf{h}_{k}\left(\mathbf{s}^{\prime}\right)$ lies in the plane of the polyhedral face $S_{k}$. Therefore, we can write

$\mathbf{h}_{k}\left(\mathbf{s}^{\prime}\right)=\left(\mathbf{s}^{\prime}-\mathbf{r}\right)_{\|} g\left(\rho_{k}, z_{k}\right)$,

where $g\left(\rho_{k}, z_{k}\right)$ is a scalar function to be found in order to satisfy the first condition given in Eq. (11). The parameters $\rho_{k}$ and $z_{k}$ read

$\rho_{k}=\left|\left(\mathbf{s}^{\prime}-\mathbf{r}\right)_{\|}\right|, \quad z_{k}=\left|Z_{k}\right|$. 
The substitution from Eq. (15) to Eq. (11) yields

$\nabla_{\mathbf{s}^{\prime}} \cdot\left(\mathbf{s}^{\prime}-\mathbf{r}\right)_{\|} g\left(\rho_{k}, z_{k}\right)=\left|\mathbf{s}^{\prime}-\mathbf{r}\right|$.

We further apply

$$
\begin{aligned}
& g\left(\rho_{k}, z_{k}\right) \nabla_{\mathbf{s}^{\prime}} \cdot\left(\mathbf{s}^{\prime}-\mathbf{r}\right)_{\|}+\left(\mathbf{s}^{\prime}-\mathbf{r}\right)_{\|} \cdot \nabla_{\mathbf{s}^{\prime}} g\left(\rho_{k}, z_{k}\right) \\
& \quad=\left|\mathbf{s}^{\prime}-\mathbf{r}\right| .
\end{aligned}
$$

Since $\nabla_{\mathbf{s}^{\prime}} \cdot\left(\mathbf{s}^{\prime}-\mathbf{r}\right)_{\|}=2$, Eq. (17) becomes

$2 g\left(\rho_{k}, z_{k}\right)+\left(\mathbf{s}^{\prime}-\mathbf{r}\right)_{\|} \cdot \nabla_{\mathbf{s}^{\prime}} g\left(\rho_{k}, z_{k}\right)=\left|\mathbf{s}^{\prime}-\mathbf{r}\right|$,

where $\left(\mathbf{s}^{\prime}-\mathbf{r}\right)_{\|} \cdot \nabla_{\mathbf{s}^{\prime}}=\rho_{k} \partial / \partial \rho_{k}$ (cf. Pohánka 1998).

Realizing that $\mathbf{s}^{\prime}-\mathbf{r}=\left(\mathbf{s}^{\prime}-\mathbf{r}\right)_{\|}+\left(\mathbf{s}^{\prime}-\mathbf{r}\right)_{\perp}=\left(\mathbf{s}^{\prime}-\mathbf{r}\right)_{\|}+$ $Z_{k} \mathbf{n}_{k}$, we define the right-hand side of Eq.(18) as follows

$\left|\mathbf{s}^{\prime}-\mathbf{r}\right|=\sqrt{\left|\left(\mathbf{s}^{\prime}-\mathbf{r}\right)_{\|}\right|^{2}+\left|Z_{k}\right|^{2}}=\sqrt{\rho_{k}^{2}+z_{k}^{2}}$.

From Eqs. (18) and (19), we get

$\frac{1}{\rho_{k}} \frac{\partial}{\partial \rho_{k}} \rho_{k}^{2} g\left(\rho_{k}, z_{k}\right)=\sqrt{\rho_{k}^{2}+z_{k}^{2}}$.

The general solution of Eq. (20) is found to be

$g\left(\rho_{k}, z_{k}\right)=\frac{1}{3 \rho_{k}^{2}}\left[\left(\rho_{k}^{2}+z_{k}^{2}\right)^{\frac{3}{2}}+c\right]$,

where $c$ is an arbitrary integration constant. However, in order to have the function $\mathbf{h}_{k}\left(\mathbf{s}^{\prime}\right)$ which satisfies the condition of the applicability of the Gauss divergence theorem (i.e. the function $\mathbf{h}_{k}\left(\mathbf{s}^{\prime}\right)$ has the integrable gradient in the whole domain $S_{k}$ ), we have to treat the singularity when $\rho_{k} \rightarrow 0$. The limit $\lim _{\rho_{k} \rightarrow 0} g\left(\rho_{k}, z_{k}\right)=0$ only if the integration constant $c=-z_{k}^{3}$. The expression in Eq. (21) then becomes

$g\left(\rho_{k}, z_{k}\right)=\frac{1}{3 \rho_{k}^{2}}\left[\left(\rho_{k}^{2}+z_{k}^{2}\right)^{\frac{3}{2}}-z_{k}^{3}\right]$.

The right-hand side of Eq. (22) is finally rearranged as follows

$$
\begin{aligned}
& g\left(\rho_{k}, z_{k}\right) \\
& =\frac{1}{3} \frac{\left(\sqrt{\rho_{k}^{2}+z_{k}^{2}}-z_{k}\right)\left(\rho_{k}^{2}+z_{k}^{2}+z_{k} \sqrt{\rho_{k}^{2}+z_{k}^{2}}+z_{k}^{2}\right)}{\rho_{k}^{2}} \\
& =\frac{1}{3}\left(\sqrt{\rho_{k}^{2}+z_{k}^{2}}+\frac{z_{k}^{2}}{\sqrt{\rho_{k}^{2}+z_{k}^{2}}+z_{k}}\right) .
\end{aligned}
$$

Substituting from Eq. (23) to Eq. (15), we get

$\mathbf{h}_{k}\left(\mathbf{s}^{\prime}\right)=\left(\mathbf{s}^{\prime}-\mathbf{r}\right)_{\|} \frac{1}{3}\left(\sqrt{\rho_{k}^{2}+z_{k}^{2}}+\frac{z_{k}^{2}}{\sqrt{\rho_{k}^{2}+z_{k}^{2}}+z_{k}}\right)$.
Consequently, the substitution from Eqs. (13), (14) and (19) to Eq. (24) yields

$$
\begin{aligned}
\mathbf{h}_{k}\left(\mathbf{s}^{\prime}\right) & \\
= & \frac{\mathbf{s}^{\prime}-\mathbf{r}-\mathbf{n}_{k} \mathbf{n}_{k} \cdot\left(\mathbf{s}^{\prime}-\mathbf{r}\right)}{3} \\
& \times\left[\left|\mathbf{s}^{\prime}-\mathbf{r}\right|+\frac{\left|\mathbf{n}_{k} \cdot\left(\mathbf{s}^{\prime}-\mathbf{r}\right)\right|^{2}}{\left|\mathbf{s}^{\prime}-\mathbf{r}\right|+\left|\mathbf{n}_{k} \cdot\left(\mathbf{s}^{\prime}-\mathbf{r}\right)\right|}\right] .
\end{aligned}
$$

From Eqs. (8) and (10), the function $H_{k}(\mathbf{r})$ becomes

$H_{k}(\mathbf{r})=\oint_{L_{k}} \mathbf{h}\left(\mathbf{l}^{\prime}\right) \cdot \mathrm{d} \xi^{\prime}$

Finally, the substitution from Eq. (25) to Eq. (26) yields

$$
\begin{aligned}
& H_{k}(\mathbf{r}) \\
& =\oint_{L_{k}} \frac{\mathbf{l}^{\prime}-\mathbf{r}-\mathbf{n}_{k} \mathbf{n}_{k} \cdot\left(\mathbf{l}^{\prime}-\mathbf{r}\right)}{3} \\
& \quad \times\left[\left|\mathbf{l}^{\prime}-\mathbf{r}\right|+\frac{\left|\mathbf{n}_{k} \cdot\left(\mathbf{l}^{\prime}-\mathbf{r}\right)\right|^{2}}{\left|\mathbf{l}^{\prime}-\mathbf{r}\right|+\left|\mathbf{n}_{k} \cdot\left(\mathbf{l}^{\prime}-\mathbf{r}\right)\right|}\right] \cdot \mathrm{d} \boldsymbol{\xi}^{\prime},
\end{aligned}
$$

where $\left|\mathbf{I}^{\prime}-\mathbf{r}\right|$ is the Euclidean spatial distance between the computation point $\mathbf{r}$ and the running integration point $\mathbf{l}^{\prime}$ on the polygon $L_{k}$. Since the vector $\mathrm{d} \xi^{\prime}$ is perpendicular to the vector $\mathbf{n}_{k}$, i.e. $\mathbf{n}_{k} \cdot \mathrm{d} \boldsymbol{\xi}^{\prime}=0$, we write

$$
\begin{aligned}
& H_{k}(\mathbf{r}) \\
& =\oint_{L_{k}} \frac{\mathbf{l}^{\prime}-\mathbf{r}}{3}\left[\left|\mathbf{l}^{\prime}-\mathbf{r}\right|+\frac{\left|\mathbf{n}_{k} \cdot\left(\mathbf{l}^{\prime}-\mathbf{r}\right)\right|^{2}}{\left|\mathbf{I}^{\prime}-\mathbf{r}\right|+\left|\mathbf{n}_{k} \cdot\left(\mathbf{l}^{\prime}-\mathbf{r}\right)\right|}\right] \cdot \mathrm{d} \xi^{\prime} .
\end{aligned}
$$

By analogy with the notation used in Pohánka (1988), the polygon segments $\left\{L_{k, l}: l=1,2, \ldots, L(k)\right\}$ form the closed polygon $L_{k}$ of the polyhedral face $S_{k} ; L(k)$ is the total number of polygon segments $L_{k, l}$ of the polyhedral face $S_{k}$. We further denote the position vectors $\mathbf{a}_{k, l}$ and $\mathbf{a}_{k, l+1}$ of the end points of the polygon segment $L_{k, l}$ (note that the vertices of the polyhedral face $S_{k}$ are numbered in the counter-clockwise sense as viewed from outside, and $\left.\mathbf{a}_{k, L(k)+1}=\mathbf{a}_{k, 1}\right)$. For every polygon segment $L_{k, l}$ we define the unit vectors $\boldsymbol{\mu}_{k, l}$ and $\boldsymbol{v}_{k, l}$. The unit vector $\boldsymbol{\mu}_{k, l}$ is parallel with the polygon segment $L_{k, l}$ and has the same orientation. It reads

$\boldsymbol{\mu}_{k, l}=\frac{\mathbf{a}_{k, l+1}-\mathbf{a}_{k, l}}{d_{k, l}}$,

where $d_{k, l}$ is the length of the segment $L_{k, l}$; i.e.,

$d_{k, l}=\left|\mathbf{a}_{k, l+1}-\mathbf{a}_{k, l}\right|$.

The unit vector $\boldsymbol{v}_{k, l}$ is perpendicular to the polygon segment $L_{k, l}$ and lies in the plane of the polyhedral face $S_{k}$. It reads

$\boldsymbol{v}_{k, l}=\boldsymbol{\mu}_{k, l} \times \mathbf{n}_{k}$. 
We define the position vector $\mathbf{I}^{\prime}$ of the point on the polygon segment $L_{k, l}$ as a function of the unit vector $\boldsymbol{\mu}_{k, l}$ in the following form

$\mathbf{I}^{\prime}=\mathbf{a}_{k, l}+\boldsymbol{\mu}_{k, l} \xi^{\prime} \quad\left(0 \leq \xi^{\prime} \leq d_{k, l}\right)$.

Similarly, we define the line element vector $\mathrm{d} \xi^{\prime}$ as a function of the unit vector $\boldsymbol{v}_{k, l}$. We have

$\mathrm{d} \boldsymbol{\xi}^{\prime}=\boldsymbol{v}_{k, l} \mathrm{~d} \xi^{\prime}$,

where $\mathrm{d} \xi^{\prime}$ is the scalar line element of the polygon segment $L_{k, l}$.

Since $\mathbf{n}_{k} \cdot \boldsymbol{\mu}_{k, l}=0$, the quantity $\mathbf{n}_{k} \cdot\left(\mathbf{I}^{\prime}-\mathbf{r}\right)=\mathbf{n}_{k} \cdot\left(\mathbf{a}_{k, l}-\mathbf{r}\right)$ in Eq. (28) neither depends on $\xi^{\prime}$ nor on the index $l$. Let us denote

$z_{k}(\mathbf{r})=\left|\mathbf{n}_{k} \cdot\left(\mathbf{l}^{\prime}-\mathbf{r}\right)\right|=\left|\mathbf{n}_{k} \cdot\left(\mathbf{a}_{k, 1}-\mathbf{r}\right)\right|$.

The line integral in Eq. (28) then takes the following form

$H_{k}(\mathbf{r})=\oint_{L_{k}} \frac{\mathbf{I}^{\prime}-\mathbf{r}}{3}\left[\left|\mathbf{I}^{\prime}-\mathbf{r}\right|+\frac{z_{k}^{2}(\mathbf{r})}{\left|\mathbf{I}^{\prime}-\mathbf{r}\right|+z_{k}(\mathbf{r})}\right] \cdot \mathrm{d} \boldsymbol{\xi}^{\prime}$.

The polygon $L_{k}$ in Eq. (35) is further rewritten as a sum of the polygon segments $L_{k, l}$. After substituting from Eqs. (32) and (33) to Eq. (35), we have

$$
\begin{aligned}
H_{k}(\mathbf{r})= & \sum_{l=1}^{L(k)} \int_{0}^{d_{k, l}} \frac{\mathbf{v}_{k, l} \cdot\left(\mathbf{a}_{k, l}-\mathbf{r}+\boldsymbol{\mu}_{k, l} \xi^{\prime}\right)}{3} \\
& \times\left[\left|\mathbf{a}_{k, l}-\mathbf{r}+\boldsymbol{\mu}_{k, l} \xi^{\prime}\right|+\frac{z_{k}^{2}(\mathbf{r})}{\left|\mathbf{a}_{k, l}-\mathbf{r}+\boldsymbol{\mu}_{k, l} \xi^{\prime}\right|+z_{k}(\mathbf{r})}\right] \mathrm{d} \xi^{\prime} .
\end{aligned}
$$

Since $\boldsymbol{v}_{k, l} \cdot \boldsymbol{\mu}_{k, l}=0$, we arrive at

$$
\begin{aligned}
H_{k}(\mathbf{r}) & =\sum_{l=1}^{L(k)} \int_{0}^{d_{k, l}} \frac{\boldsymbol{v}_{k, l} \cdot\left(\mathbf{a}_{k, l}-\mathbf{r}\right)}{3} \\
\times & {\left[\left|\mathbf{a}_{k, l}-\mathbf{r}+\boldsymbol{\mu}_{k, l} \xi^{\prime}\right|+\frac{z_{k}^{2}(\mathbf{r})}{\left|\mathbf{a}_{k, l}-\mathbf{r}+\boldsymbol{\mu}_{k, l} \xi^{\prime}\right|+z_{k}(\mathbf{r})}\right] \mathrm{d} \xi^{\prime} . }
\end{aligned}
$$

We decompose the vector $\mathbf{a}_{k, l}-\mathbf{r}$ in Eq. (36) into the vector components $\boldsymbol{\mu}_{k, l}, \boldsymbol{v}_{k, l}$ and $\mathbf{n}_{k}$, and adopt the following notation (cf. Pohánka 1998, Eqs. 28 and 29)

$$
\begin{aligned}
u_{k, l}(\mathbf{r}) & =\boldsymbol{\mu}_{k, l} \cdot\left(\mathbf{a}_{k, l}-\mathbf{r}\right), \\
w_{k, l}(\mathbf{r}) & =\boldsymbol{v}_{k, l} \cdot\left(\mathbf{a}_{k, l}-\mathbf{r}\right), \\
Z_{k}(\mathbf{r}) & =\mathbf{n}_{k} \cdot\left(\mathbf{a}_{k, 1}-\mathbf{r}\right) .
\end{aligned}
$$

From Eqs. (32) and (37), we write

$$
\begin{aligned}
\left|\mathbf{I}^{\prime}-\mathbf{r}\right| & =\left|\mathbf{a}_{k, l}-\mathbf{r}+\boldsymbol{\mu}_{k, l} \xi^{\prime}\right| \\
& =\sqrt{\left(u_{k, l}(\mathbf{r})+\xi^{\prime}\right)^{2}+w_{k, l}^{2}(\mathbf{r})+z_{k}^{2}(\mathbf{r}) .}
\end{aligned}
$$

The substitution from Eqs. (37) and (38) to Eq. (36) yields

$$
\begin{aligned}
H_{k}(\mathbf{r})= & \sum_{l=1}^{L(k)} \int_{0}^{d_{k, l}} \frac{w_{k, l}(\mathbf{r})}{3} \\
& \times\left[\sqrt{\left(u_{k, l}(\mathbf{r})+\xi^{\prime}\right)^{2}+w_{k, l}^{2}(\mathbf{r})+z_{k}^{2}(\mathbf{r})}\right. \\
& \left.+\frac{z_{k}^{2}(\mathbf{r})}{\sqrt{\left(u_{k, l}(\mathbf{r})+\xi^{\prime}\right)^{2}+w_{k, l}^{2}(\mathbf{r})+z_{k}^{2}(\mathbf{r})}+z_{k}(\mathbf{r})}\right] \mathrm{d} \xi^{\prime} .
\end{aligned}
$$

Furthermore, after applying the substitution $u_{k, l}(\mathbf{r})+\xi^{\prime}=$ $\xi$, we get

$$
\begin{aligned}
H_{k}(\mathbf{r})= & \sum_{l=1}^{L(k)} \int_{u_{k, l}}^{v_{k, l}} \frac{w_{\mathrm{k}, 1}(\mathbf{r})}{3}\left[\sqrt{\xi^{2}+w_{k, l}^{2}(\mathbf{r})+z_{k}^{2}(\mathbf{r})}\right. \\
& \left.+\frac{z_{k}^{2}(\mathbf{r})}{\sqrt{\xi^{2}+w_{k, l}^{2}(\mathbf{r})+z_{k}^{2}(\mathbf{r})}+z_{k}(\mathbf{r})}\right] \mathrm{d} \xi,
\end{aligned}
$$

where

$v_{k, l}(\mathbf{r})=u_{k, l}(\mathbf{r})+d_{k, l}$.

Denoting

$\Phi(u, v, w, z)=\int_{u}^{v} \frac{w}{\sqrt{\xi^{2}+w^{2}+z^{2}}+z} \mathrm{~d} \xi$,

$\Phi_{3}(u, v, w, z)=\int_{u}^{v} w \sqrt{\xi^{2}+w^{2}+z^{2}} \mathrm{~d} \xi$,

we finally arrive at

$$
\begin{aligned}
H_{k}(\mathbf{r})= & \frac{1}{3} \sum_{l=1}^{L(k)}\left\{z_{k}^{2}(\mathbf{r}) \Phi\left(u_{k, l}(\mathbf{r}), v_{k, l}(\mathbf{r}), w_{k, l}(\mathbf{r}), z_{k}(\mathbf{r})\right)\right. \\
& \left.+\Phi_{3}\left(u_{k, l}(\mathbf{r}), v_{k, l}(\mathbf{r}), w_{k, l}(\mathbf{r}), z_{k}(\mathbf{r})\right)\right\}
\end{aligned}
$$

The closed analytical expressions for $\Phi$ read (Pohánka 1988, 1998)

$\Phi(u, v, w, z)=w L(u, v, w, z)+2 z A(u, v, w, z)$,

integrating the right-hand side of Eq. (42), we get

$$
\begin{aligned}
& \Phi_{3}(u, v, w, z) \\
& =\frac{w}{4}(v-u)\left[\frac{(v+u)^{2}}{T(u, v, w, z)}+T(u, v, w, z)\right] \\
& \quad+\frac{w}{2}\left(w^{2}+z^{2}\right) L(u, v, w, z),
\end{aligned}
$$


where

$L(u, v, w, z)=\ln \frac{\sqrt{v^{2}+w^{2}+z^{2}}+v}{\sqrt{u^{2}+w^{2}+z^{2}}+u}$,

$A(u, v, w, z)$

$$
=-\arctan \frac{2 w(v-u)}{T(u, v, w, z)^{2}-(v-u)^{2}+2 z T(u, v, w, z)},
$$

$T(u, v, w, z)=\sqrt{u^{2}+w^{2}+z^{2}}+\sqrt{v^{2}+w^{2}+z^{2}}$.

\section{The optimum expression for the potential}

By analogy with Pohánka (1988, 1998), we optimize the expression for the potential by reducing the number of logarithm and arctangent terms, treating the undefined operations (e.g. expressions of the type 0/0), and improving the computational accuracy. The optimum expression for numerical calculation of the vector function $\mathbf{G}_{k}(\mathbf{r})$ in Eq. (9) was derived by (Pohánka 1998, Eqs. 33, 34 and 35) in the following form

$$
\begin{aligned}
\mathbf{G}_{k}(\mathbf{r})= & \sum_{l=1}^{L(k)}\left\{\Phi_{2}\left(u_{k, l}(\mathbf{r}), v_{k, l}(\mathbf{r}), w_{k, l}(\mathbf{r}), Z_{k}(\mathbf{r})\right) \boldsymbol{v}_{k, l}\right. \\
& \left.+Z_{k}(\mathbf{r}) \Phi\left(u_{k, l}(\mathbf{r}), v_{k, l}(\mathbf{r}), w_{k, l}(\mathbf{r}), z_{k}(\mathbf{r})\right) \mathbf{n}_{k}\right\},
\end{aligned}
$$

where the expression for $\Phi_{2}$ reads (Pohánka 1998)

$$
\begin{aligned}
& \Phi_{2}(u, v, w, Z) \\
& =\frac{1}{4}(v-u)\left[\frac{(v+u)^{2}}{T(u, v, w, z)}+T(u, v, w, z)\right] \\
& \quad+\frac{1}{2}\left(w^{2}+z^{2}\right) L(u, v, w, z) .
\end{aligned}
$$

Comparing the expressions for $\Phi_{3}$ and $\Phi_{2}$ in Eqs. (45) and (50), the following relation between them is obtained

$\Phi_{3}(u, v, w, z)=w \Phi_{2}(u, v, w, Z)$.

Utilizing Eq. (51), the expression for the scalar function $H_{k}(\mathbf{r})$ in Eq. (43) becomes

$$
\begin{aligned}
H_{k}(\mathbf{r})= & \frac{1}{3} \sum_{l=1}^{L(k)}\left\{z_{k}^{2}(\mathbf{r}) \Phi\left(u_{k, l}(\mathbf{r}), v_{k, l}(\mathbf{r}), w_{k, l}(\mathbf{r}), z_{k}(\mathbf{r})\right)\right. \\
& \left.+w_{k, l}(\mathbf{r}) \Phi_{2}\left(u_{k, l}(\mathbf{r}), v_{k, l}(\mathbf{r}), w_{k, l}(\mathbf{r}), Z_{k}(\mathbf{r})\right)\right\}
\end{aligned}
$$

The vectors $\boldsymbol{\mu}_{k, l}$ and $\boldsymbol{v}_{k, l}$ are perpendicular to $\mathbf{n}_{k}$, i.e. $\mathbf{n}_{k}$. $\boldsymbol{\mu}_{k, l}=0$ and $\mathbf{n}_{k} \cdot \boldsymbol{v}_{k, l}=0$. From Eq. (50), it also follows that $\Phi_{2}\left(u_{k, l}(\mathbf{r}), v_{k, l}(\mathbf{r}), w_{k, l}(\mathbf{r}), Z_{k}(\mathbf{r})\right)=\Phi_{2}\left(u_{k, l}(\mathbf{r}), v_{k, l}(\mathbf{r})\right.$, $\left.w_{k, l}(\mathbf{r}), z_{k}(\mathbf{r})\right)$. The substitution of the functions $\mathbf{G}_{k}(\mathbf{r})$ and $H_{k}(\mathbf{r})$ from Eqs. (49) and (52) to Eq. (9) then yields

$$
\begin{aligned}
V(\mathbf{r})=\mathrm{G} \sum_{k=1}^{K}\left[\sum_{l=1}^{L(k)}\left(\frac{\rho_{0}+\boldsymbol{\rho}_{\mathbf{1}} \cdot \mathbf{r}}{2} Z_{k}(\mathbf{r})+\frac{\boldsymbol{\rho}_{\mathbf{1}} \cdot \mathbf{n}_{k}}{3} z_{k}^{2}(\mathbf{r})\right)\right. \\
\quad \times \Phi\left(u_{k, l}(\mathbf{r}), v_{k, l}(\mathbf{r}), w_{k, l}(\mathbf{r}), z_{k}(\mathbf{r})\right) \\
+\sum_{l=1}^{L(k)} \frac{\boldsymbol{\rho}_{\mathbf{1}} \cdot \mathbf{n}_{k}}{3} w_{k, l}(\mathbf{r}) \Phi_{2}\left(u_{k, l}(\mathbf{r}), v_{k, l}(\mathbf{r}),\right. \\
\left.\left.\quad \times w_{k, l}(\mathbf{r}), z_{k}(\mathbf{r})\right)\right] .
\end{aligned}
$$

As seen from Eq. (9) only those components of the function $\mathbf{G}_{k}(\mathbf{r})$ contribute to the potential which are parallel to the surface normal (i.e. the components along the unit normal vector $\mathbf{n}_{k}$ ).

The expression for the potential in Eq. (53) has very close resemblances with the formula for the attraction derived by (Pohánka, 1998, Eq. 52). To obtain the final form of Eq. (53) in the same way as Pohánka proposed by introducing a small positive number $\varepsilon$ in order to avoid any undefined operations when the computation point is near to the surface of the polyhedral body, cf. Pohánka $(1988,1998)$, the functions $\Phi(u, v, w, z)$ and $\Phi_{2}(u, v, w, z)$ are replaced by the functions $\Phi(u, v, w, z, \varepsilon)$ and $\Phi_{2}(u, v, w, z, \varepsilon)$, respectively. From Eq. (53), we have

$$
\begin{aligned}
& V(\mathbf{r}, \varepsilon) \\
& =\mathrm{G} \sum_{k=1}^{K}\left[\sum_{l=1}^{L(k)}\left(\frac{\rho_{0}+\boldsymbol{\rho}_{\mathbf{1}} \cdot \mathbf{r}}{2} z_{k}(\mathbf{r})+\frac{\boldsymbol{\rho}_{\mathbf{1}} \cdot \mathbf{n}_{k}}{3} z_{k}^{2}(\mathbf{r})\right)\right. \\
& \quad \times \Phi\left(u_{k, l}(\mathbf{r}), v_{k, l}(\mathbf{r}), w_{k, l}(\mathbf{r}), z_{k}(\mathbf{r}), \varepsilon\right) \\
& \quad+\sum_{l=1}^{L(k)} \frac{\boldsymbol{\rho}_{\mathbf{1}} \cdot \mathbf{n}_{k}}{3} w_{k, l}(\mathbf{r}) \Phi_{2}\left(u_{k, l}(\mathbf{r}), v_{k, l}(\mathbf{r})\right. \\
& \left.\left.\quad \times w_{k, l}(\mathbf{r}), z_{k}(\mathbf{r}), \varepsilon\right)\right]
\end{aligned}
$$

where

$$
\begin{aligned}
& \Phi(u, v, w, z, \varepsilon)= w L(u, v, w, z+\varepsilon) \\
&+2 z A(u, v, w, z+\varepsilon), \\
& \Phi_{2}(u, v, w, z, \varepsilon) \\
&=\left.\frac{1}{4}(v-u)\left[\frac{(v+u)^{2}}{T(u, v, w, z+\varepsilon)}+T u, v, w, z+\varepsilon\right)\right] \\
& \quad+\frac{1}{2}\left(w^{2}+z^{2}\right) L(u, v, w, z+\varepsilon) .
\end{aligned}
$$

The appropriate choice of the parameter $\varepsilon$ is discussed in detail in Pohánka (1988, 1998). 


\section{The algorithm of calculation}

For an efficient calculation of the potential given in Eq. (54) we adopted the same optimum algorithm as proposed by Pohánka (1998). The input parameters are: the value of density $\rho_{0}$ at a suitably chosen origin of the local coordinate system used for a description of the density model within the volume of the polyhedral body (we note that this coordinate origin can be located either inside or outside of the polyhedral body), the gradient of a linear density distribution function $\boldsymbol{\rho}_{\mathbf{1}}$, the total number of polyhedral faces $K$, and the total number of polygon segments $L(k)$ given for every polyhedral face $S_{k}$. For every vertex of the polyhedral face $S_{k}$, we compute the radius vector $\mathbf{a}_{k, l}$. As stated previously, the vertices of the polyhedral face $S_{k}$ are numbered in the counter-clockwise sense as viewed from outside, and $\mathbf{a}_{k, L(k)+1}=\mathbf{a}_{k, 1}$. The last input is the radius vector $\mathbf{r}$ of the computation point. We note here that the expression in Eq. (54) for the potential holds for any computation point outside and on the surface of the polyhedral body.

The computation is then realized as follows: We compute the lengths $\left\{d_{k, l}: l=1,2, \ldots, L(k) ; k=1,2, \ldots, K\right\}$ of the polygon segments $L_{k, l}$, and the corresponding unit vectors $\left\{\boldsymbol{\mu}_{k, l}: l=1,2, \ldots, L(k) ; k=1,2, \ldots, K\right\}$ according to the following equations (cf. Eqs. (29), (30))

$d_{k, l}=\left|\mathbf{a}_{k, l+1}-\mathbf{a}_{k, l}\right|, \quad \boldsymbol{\mu}_{k, l}=\frac{\mathbf{a}_{k, l+1}-\mathbf{a}_{k, l}}{d_{k, l}}$.

For every polyhedral face $\left\{S_{k}: k=1,2, \ldots, K\right\}$ we compute the unit normal vector $\mathbf{n}_{k}$ oriented outwards from the polyhedral face $S_{k}$ using the following expression:

$\mathbf{n}_{k}=\frac{\mathbf{N}_{k}}{\left|\mathbf{N}_{k}\right|}$,

where

$\mathbf{N}_{k}=\sum_{l=2}^{L(k)-1}\left(\mathbf{a}_{k, l}-\mathbf{a}_{k, 1}\right) \times\left(\mathbf{a}_{k, l+1}-\mathbf{a}_{k, 1}\right)$.

For all polygon segments $\left\{L_{k, l}: l=1,2, \ldots, L(k)\right\}$ of the polyhedral face $S_{k}$ we compute the normal unit vectors $\left\{\boldsymbol{v}_{k, l}: l=1,2, \ldots, L(k)\right\}$ using Eq. (31); i.e.,

$\boldsymbol{v}_{k, l}=\boldsymbol{\mu}_{k, l} \times \mathbf{n}_{k}$.

We further compute the parameters $u_{k, l}, v_{k, l}, w_{k, l}, Z_{k}$ and $z_{k}$ which depend on the position vector $\mathbf{r}$ of the computation point. With reference to Eqs. (37) and (40), we have

$$
\begin{aligned}
u_{k, l} & =\boldsymbol{\mu}_{k, l} \cdot\left(\mathbf{a}_{k, l}-\mathbf{r}\right), \\
v_{k, l} & =u_{k, l}+d_{k, l}, \\
w_{k, l} & =\boldsymbol{v}_{k, l} \cdot\left(\mathbf{a}_{k, l}-\mathbf{r}\right), \\
Z_{k} & =\mathbf{n}_{k} \cdot\left(\mathbf{a}_{k, 1}-\mathbf{r}\right), \quad z_{k}=\left|Z_{k}\right| .
\end{aligned}
$$

For the sake of completeness we recapitulate the computation steps given by Pohánka (1998). For the given numbers $u, v(v=u+d, d>0), w, z(z \geq 0)$ and the parameter $\varepsilon$, the computation of the functions $\Phi(u, v, w$, $z, \varepsilon)$ and $\Phi_{2}(u, v, w, z, \varepsilon)$ in Eqs. (55) and (56) is carried out in the following consecutive steps:

(a) $z_{\varepsilon}=z+\varepsilon$,

(b) $W^{2}=w^{2}+z^{2}, W_{\varepsilon}^{2}=w^{2}+z_{\varepsilon}^{2}$,

(c) $U_{\varepsilon}=\sqrt{u^{2}+W_{\varepsilon}^{2}}, V_{\varepsilon}=\sqrt{v^{2}+W_{\varepsilon}^{2}}$,

(d) $T_{\varepsilon}=U_{\varepsilon}+V_{\varepsilon}$

(e) $\operatorname{sign}(u)=\operatorname{sign}(v): L_{\varepsilon}=\operatorname{sign}(v) \ln \frac{V_{\varepsilon}+|v|}{U_{\varepsilon}+|u|}, \operatorname{sign}(u) \quad \neq$ $\operatorname{sign}(v): L_{\varepsilon}=\ln \frac{\left(V_{\varepsilon}+|v|\right)\left(U_{\varepsilon}+|u|\right)}{W_{\varepsilon}^{2}}$,

(f) $A_{\varepsilon}=-\arctan \frac{2 w d}{\left(T_{\varepsilon}+d\right)\left|T_{\varepsilon}-d\right|+2 T_{\varepsilon} z_{\varepsilon}}$.

(g) $\Phi(u, v, w, z, \varepsilon)=w L_{\varepsilon}+2 z A_{\varepsilon}$,

$$
\Phi_{2}(u, v, w, z, \varepsilon)=\frac{1}{4} d\left[\frac{(v+u)^{2}}{T_{\varepsilon}}+T_{\varepsilon}\right]+\frac{1}{2} W^{2} L_{\varepsilon} .
$$

Finally, the potential $V(\mathbf{r}, \varepsilon)$ is obtained from Eq. (54).

\section{Concluding remarks}

We have derived the analytical formula by means of line integrals for the gravitational potential of an arbitrary polyhedral body having a linearly varying density. The corresponding analytical formula for the Cartesian coordinate components of the gravitational attraction vector was given by Pohánka (1998). As seen from Eq. (9), the derivation of the gravitational potential was reduced to finding only the closed analytical solution of the surface integral in the scalar function $H_{k}(\mathbf{r})$, while the solution of the surface integral in the vector function $\mathbf{G}_{k}(\mathbf{r})$ was already derived in Pohánka (1998). We further adopted the optimized expressions from Pohánka $(1988,1998)$ in forming the optimal expression for the gravitational potential by reducing the number of logarithm and arctangent terms, treating the undefined operations, and improving the precision of numerical operations when the computation point is far away from the polyhedral body. Finally, we adopted the optimum algorithm as proposed by Pohánka (1998) for computing the gravitational potential. The optimum expressions and uniform algorithm for computing the gravitational potential and attraction are numerically very simple and they are valid for any point outside and on the surface of the polyhedral body.

The main advantage of using the line integral approach in detailed local gravity field modelling is that it can be utilized for any irregular digital terrain and density models without 
additional data pre-processing. The analytical expressions for volume elements with linearly varying density distribution models improve the numerical efficiency in the forward modelling of the gravitational field of geological structures with the variable density distribution such as sedimentary basins where the density increases with depth due to compaction. Moreover, they improve to some extent the numerical efficiency when used for modelling the gravitational field of inhomogeneous density structures which can accurately be approximated by the pricewise linear density model.

Acknowledgments We thank V. Pohánka (Geophysical Institute of the Slovak Academy of Sciences) and two anonymous reviewers for their constructive comments.

Open Access This article is distributed under the terms of the Creative Commons Attribution Noncommercial License which permits any noncommercial use, distribution, and reproduction in any medium, provided the original author(s) and source are credited.

\section{References}

Artemjev ME, Kaban MK, Kucherinenko VA, Demjanov GV, Taranov VA (1994) Subcrustal density inhomogeneities of the Northern Euroasia as derived from the gravity data and isostatic models of the lithosphere. Tectonophysics 240:248-280

Barnett (1976) Theoretical modeling of the magnetic and gravitational fields of an arbitrary shaped three dimensional body. Geophysics 41:1353-1364

Bessel FW (1813) Auszug aus einem Schreiben des Herrn Prof. Bessel. Zach's Monatliche Correspondenz zur Beförderung der Erd- und Himmelskunde XXVII:80-85

Chai Y, Hinze WJ (1988) Gravity inversion of an interface above which the density contrast varies exponentially with depth. Geophysics 53:837-845

Collette BI (1965) Chart for determining the gravity effect of two and three dimensional bodies bounded by arbitrary polygons. Geophys Prospect 13:12-21

Everest G (1830) An account of the measurement of the arc of the meridian between the parallels of $18^{\circ} 3^{\prime}$ and $24^{\circ} 7^{\prime}$. The Royal Society, Printed by JL Cox, London

Gallardo-Delgado LA, Perez-Flores MA, Gomez-Trevino E (2003) A versatile algorithm for joint inversion of gravity and magnetic data. Geophysics 68:949-959

García-Abdeslem J (1992) Gravitational attraction of a rectangular prism with depth dependent density. Geophysics 57:470-473

García-Abdeslem J (2005) Gravitational attraction of a rectangular prism with density varying with depth following a cubic polynomial. Geophysics 70:J39-J42

Götze HJ, Lahmeyer B (1988) Application of three-dimensional interactive modelling in gravity and magnetics. Geophysics 53(8):1096-1108

Hansen RO (1999) An analytical expression for the gravity field of a polyhedral body with linearly varying density. Geophysics 64(1):75-77

Holstein H, Ketteridge B (1996) Gravimetric analysis of uniform polyhedra. Geophysics 61(2):357-364
Holstein H, Schürholz P, Starr AJ, Chakraborty M (1999) Comparison of gravimetric formulas for uniform polyhedra. Geophysics 64(5): 1434-1446

Holstein H (2002) Gravimagnetic similarity in anomaly formulas for uniform polyhedra. Geophysics 67(4):1126-1133

Holstein H (2002) Invariance in gravimagnetic anomaly formulas for uniform polyhedra. Geophysics 67(4):1134-1137

Holstein H (2003) Gravimagnetic anomaly formulas for polyhedra of spatially linear media. Geophysics 68(1):157-167

Hurbbert MK (1948) A line integral method of computing the gravity effects of two dimensional masses. Geophysics 13:215-225

Ivan M (1990) Comment on "Optimum expression for computation of the gravity field of homogeneous polyhedral body" by V. Pohánka. Geophys Prospect 38:331-332

Kwok Y-K (1991) Gravity gradient tensor due to a polyhedron with polygonal facets. Geophys Prospect 39:435-443

Mader K (1951) Das Newtonsche Raumpotential prismatischer Körper und seine Ableitungen bis zur dritten Ordnung. Sonderheft 11 der Österreichischen Zeitschrift für Vermessungswesen. Österreichischer Verein für Vermessungswesen, Wien

Mollweide KB (1813) Auflösung einiger die Anziehing von Linien Flächen und Köpern betreffenden Aufgaben unter denen auch die in der Monatl Corresp Bd XXIV. S, 522. vorgelegte sich findet. Zach's Monatliche Correspondenz zur Beförderung der Erd- und Himmelskunde, Bd XXVII:26-38

Nagy D, Papp G, Benedek J (2000) The gravitational potential and its derivatives for the prism. J Geod 74(7/8):552-560

Okabe M (1979) Analytical expressions for gravity anomalies due to homogeneous polyhedral bodies and translation into magnetic anomalies. Geophysics 44(4):730-741

Paul MK (1974) The gravity effect of a homogeneous polyhedron for three dimensional interpretation. Pure Appl Geophys 112:553-561

Petrović S (1996) Determination of the potential of homogeneous polyhedral bodies using line integrals. J Geod 71:44-52

Pohánka V (1988) Optimum expression for computation of the gravity field of a homogeneous polyhedral body. Geophys Prospect 36:733-751

Pohánka V (1990) Replay to comment by M. Ivan. Geophys Prospect 38:333-335

Pohánka V (1998) Optimum expression for computation of the gravity field of a polyhedral body with linearly increasing density. Geophys Prospect 46:391-404

Takin M, Talwani M (1966) Rapid computation of gravitational attraction of topography on a spherical earth. Geophys Prospect 14:119142

Talwani M, Worzel JL, Landisman M (1959) Rapid gravity computation for two dimension bodies with application to the Mendocino submarine fracture zone. J Geophys Res 64:49-59

Talwani M, Ewing M (1960) Rapid computation of gravitational attraction of three dimensional bodies of arbitrary shape. Geophysics 25:203-225

Tsoulis D, Petrović S (2001) On the singularities of the gravity field of a homogeneous polyhedral body. Geophysics 66:535-539

Werner RA, Scheeres DJ (1997) Exterior gravitation of polyhedron derived and compared with harmonic and mascon gravitation representations of asteroid 4769 Castalia. Celest Mech Dyn Astron 65:313-344

Zach (1811) Zach's Monatliche Correspondenz zur Beförderung der Erd- und Himmelskunde, Bd XXVII: 522 